\title{
Alveolar Epithelial Damage A Critical Difference between High Pressure and Oleic Acid-induced Low Pressure Pulmonary Edema
}

\author{
J. S. G. Montaner, J. Tsang, K. G. Evans, J. B. M. Mullen, A. R. Burns, D. C. Walker, B. Wiggs, and J. C. Hogg
}

University of British Columbia, Pulmonary Research Laboratory, St. Paul's Hospital, Vancouver, British Columbia V6Z 1 Y6 Canada

\begin{abstract}
The present study was designed to compare high pressure pulmonary edema (HPPE) and oleic acid-induced low pressure pulmonary edema (OAPE) in dogs when similar amounts of extra vascular water were present in the lung. The high pressure edema was produced by intravenous fluid overload and by inflating an aortic balloon catheter $(n=6)$. The low pressure edema was produced by the injecting $0.08 \mathrm{mg} / \mathrm{kg}$ oleic acid suspended in 5 $\mathrm{ml}$ saline $(n=6)$. Comparison of the difference between initial control measurements and final measurements in the edematous states showed that the animals with OAPE had a greater fall in percent oxygen saturation and a greater increase in shunt fractions. The light microscopic studies showed that OAPE was associated with greater amounts of alveolar flooding than HPPE where the edema fluid was located to a greater extent in the peribronchial interstitial space. The electron microscopy studies showed that the alveolar flooding in OAPE was associated with epithelial disruption, and tracer studies carried out in rabbits showed that dextran $(150,000$ mol wt) could pass from blood to airspace and that dextran $(40,000 \mathrm{~mol} w \mathrm{wt})$ could pass from airspace to blood in OAPE. We conclude that epithelial disruption is responsible for the excessive alveolar flooding in OAPE and that this results in a greater impairment in gas exchange.
\end{abstract}

\section{Introduction}

In a study of fluid accumulation in high pressure and alloxaninduced low pressure pulmonary edema, Staub et al. (1) reported that edema fluid first accumulated in the interstitial compartment around the large vessel before it flooded the airspace. In a subsequent study Vreim and Staub (2) also showed that in alloxaninduced low pressure pulmonary edema (LPPE), ${ }^{1}$ alveolar flooding occurs more readily and the fluid has a higher protein content and an increased number of cells compared with high pressure pulmonary edema (HPPE) (3). These observations suggest that epithelial permeability may be quite different under

\section{Address correspondence to Dr. Hogg. 1986. \\ Received for publication 26 July 1985 and in revised form 6 February}

1. Abbreviations used in this paper: ARDS, adult respiratory distress syndrome; BP, blood pressure; CO, cardiac output; EVLW, extravascular lung water per gram of blood-free dry lung; FITC, fluorescein isothiocyanate; HPPE, high pressure pulmonary edema; LPPE, low pressure pulmonary edema; OAPE, oleic acid-induced low pressure pulmonary edema; $P_{L}$, transpulmonary pressure; $P$ pa, pulmonary artery pressure; Ppaw, pulmonary wedge pressure; Qs/Qt, shunt fractions; RA, right atrium; SAT, percent oxygen saturation; V/Q, ventilation-perfusion.

J. Clin. Invest.

(C) The American Society for Clinical Investigation, Inc. 0021-9738/86/06/1786/11 $\$ 1.00$

Volume 77, June 1986, 1786-1796 the two conditions, and this is supported by the observations that HPPE clears rapidly with therapy (4) compared with LPPE, which takes days to resolve (5). This study was designed to determine the nature of the epithelial disruption in the early stages of oleic acid-induced low pressure pulmonary edema (OAPE) and whether this is associated with a different distribution of edema fluid in the lung. The studies show that with similar amounts of edema present there was greater alveolar flooding and less interstitial edema in OAPE than in HPPE. Electron microscopy and tracer studies show greater disruption of the epithelium in OAPE. The physiological data show a greater deterioration in gas exchange with OAPE, particularly when differences in cardiac output (CO) are taken into account.

\section{Methods}

Physiological studies. Mongrel dogs weighing $15.6 \pm 1.2 \mathrm{~kg}$ were anesthetized with pentobarbital sodium $(25-30 \mathrm{mg} / \mathrm{kg})$, placed in a supine position, and intubated with a cuffed endotracheal tube. They were ventilated with a Harvard animal ventilator at a tidal volume of $12-15 \mathrm{ml} /$ $\mathrm{kg}$ and a respiratory rate of $10-15$ per min where the inspired gas was $100 \%$ oxygen during the entire experimental procedure. A 7 Fr double lumen Swan-Ganz, thermistor-tipped catheter (702-027 Edwards Laboratory, Sandusky, $\mathrm{OH}$ ) was placed in the pulmonary artery under fluoroscopic control for the measurements of pulmonary artery pressure (Ppa), pulmonary wedge pressure (Ppaw), and $\mathrm{CO}$ by the thermodilution technique. A multiple side-hole catheter was placed into the right atrium (RA) and a balloon-tipped catheter was placed in the intrathoracic portion of the descending aorta via one of the carotid arteries. A catheter was placed in the descending aorta, proximal to the balloon catheter, for measurements of the arterial blood pressure (BP), and a catheter was placed in a femoral vein to infuse fluids. The ureters were ligated to minimize diuresis during the experiments. All the pressures were referred to the midchest.

Vascular pressures were recorded using an 8 channel recorder (model 77528, Hewlett Packard Co., Palo Alto, CA). Mixed venous and arterial blood was sampled simultaneously for blood gas analysis (Radiometer ABL 2, Denmark, Copenhagen). After CO was measured, albumin macroaggregates labeled with ${ }^{125}$ I were injected into the right atrium to mark regional pulmonary blood flow (6). When these initial measurements were completed the animals were divided into three groups. Group I $(n=6)$ served as a control and the animals were monitored for $2 \mathrm{~h}$ without further intervention. HPPE was produced in group II $(n=6)$ by inflating the balloon-tipped catheter in the aorta and by infusing $0.9 \%$ saline solution equivalent to $25 \%$ of body weight over $2 \mathrm{~h}$. Ppa was kept within a range of $35-45 \mathrm{~cm} \mathrm{H}_{2} \mathrm{O}$ by varying the inflation of the aortic balloon to modify the cardiac afterload. With the ongoing infusion of fluid and the inflation of the aortic balloon, systemic BP ranged between 150 and $200 \mathrm{mmHg}$. An experimental model of LPPE was produced in group III $(n=6)$ by injecting oleic acid $(0.08 \mathrm{mg} / \mathrm{kg}$ suspended in $5 \mathrm{ml}$ of $0.9 \%$ saline) (7) in a bolus through the multiple side-hole catheter in the right atrium. As $\mathrm{CO}$ tended to fall in oleic acid pulmonary edema, and tended to increase or remain the same in HPPE, two additional experiments were performed where OAPE was produced and arteriovenous shunts were opened in the groin to increase $\mathrm{CO}$ to levels comparable with that seen in HPPE.

After $2 \mathrm{~h}$ the measurements of Ppa, Ppaw, BP, mixed venous and 
arterial blood gases were repeated in all three groups. After measuring $\mathrm{CO}$, albumin macroaggregates labeled with ${ }^{99 \mathrm{~m}} \mathrm{Tc}$ were injected into the right atrium as a second blood flow marker. Autologus red blood cells labelled with ${ }^{51} \mathrm{Cr}(8)$ were then injected to mark the intravascular space. Four $5-\mathrm{cm}^{3}$ blood samples were obtained $5 \mathrm{~min}$ later and the animals were killed with a bolus of saturated KCL in the left ventricle. The chest was opened and the pulmonary vessels ligated while the lungs were kept inflated with $3 \mathrm{~cm} \mathrm{H}_{2} \mathrm{O}$ positive end expiratory pressure. The trachea was then clamped and the lungs were removed and inflated to a trans-

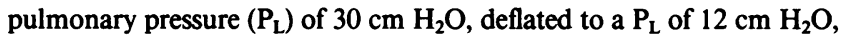
and then frozen over liquid nitrogen vapor. After freezing, the lungs were separated and cut into $2-\mathrm{cm}$ slices from the dorsal (dependant) to the ventral surface. Each slice was divided into small samples that were placed in preweighed vials. These vials were then reweighed to obtain the wet weight of the sample and counted in a gamma scintillation counter (Gamma 7000, Beckman Instruments, Inc., Fullerton, CA) for all three isotopes with appropriate corrections for overlap, decay, and background. The vials were dried in an oven $\left(60^{\circ} \mathrm{C}\right)$ to a constant weight to obtain the dry weight of the sample.

Shunt fractions $(\mathrm{Qs} / \mathrm{Qt})$ were calculated using standard equations (9). $\mathrm{Qs} / \mathrm{Qt}=(\mathrm{Cc}-\mathrm{Ca}) /(\mathrm{Cc}-\mathrm{Cv}) \times 100 \%$, where $\mathrm{Cc}, \mathrm{Ca}$, and $\mathrm{Cv}$ are the oxygen contents of the pulmonary capillary blood, the arterial blood, and the central venous blood, respectively, with the inspired oxygen at $100 \%$. The oxygen content of each sample can be calculated by measuring the oxygen tension, the percentage of oxygen saturation, and the amount of hemoglobin in each sample. Extravascular lung water per gram of blood free dry lung (EVLW) was calculated using the measurements of wet weight and dry weight and ${ }^{51} \mathrm{Cr}$-counts in each sample as previously described (10). The result was expressed in grams of water per gram of blood-free dry tissue. Regional blood flow was obtained by using the measurements of $\mathrm{CO}$ and the ${ }^{125} \mathrm{I}-$ or ${ }^{99 \mathrm{~m}} \mathrm{Tc}$-counts in each sample (6), calculated as follows: regional blood flow $(\mathrm{ml} / \mathrm{min})=($ radioactive counts in sample $\times \mathrm{CO}) /($ total radioactive counts in both lungs $) \times(\mathrm{ml} / \mathrm{min})$.

The data concerning vascular pressures, cardiac outputs, pulmonary vascular resistances, blood gases, and Qs/Qt within each group were analyzed by comparing the initial to the final values using a $t$ test for paired data. The differences between the groups were analyzed by comparing the change from initial to final value by using an analysis of variance. The data concerning the regional blood flows to each lung slice before and after the development of edema were compared using a $t$ test for paired data. The change in regional blood flow $\left(\Delta Q_{R}\right)$ after edema formation was correlated to the EVLW in each sample by using linear regression analysis.

Light microscopic studies. Tissues from one control and two of each type of experimental animal were examined by light microscopy. Random samples taken from the frozen lung slices were placed in Carnoy's solution that was precooled to $-70^{\circ} \mathrm{C}$ in dry ice. After $24 \mathrm{~h}$, the samples were gradually rewarmed to room temperature over a period of $6 \mathrm{~d}$. The samples were processed for histology in the usual manner and sections were stained with hematoxylin and eosin. A minimum of 25 and a maximum of 35 slides were prepared from each animal and coded before examination by two observers. These slides were sorted into those that were normal, those with only interstitial edema, and those with both alveolar flooding and interstitial edema. A chi-square analysis (11) was used to test for the differences in the distribution of edema fluid between the interstitial and alveolar spaces in the two treatment groups. A difference at the level of $P<0.05$ was accepted as significant. The remainder of the lung not used for histology was divided into multiple samples and used to calculate extra vascular water, which was expressed as mean \pm SD.

The same coded slides were also examined using a microscope equipped with a camera lucida so that the histological features of the vessel and cuff areas could be traced on the digitizing board of an Apple computer. The internal and external areas of 1,836 vessels and the areas of the cuff of interstitial space were traced and recorded. The presence or absence of hemorrhage into the cuff was also noted. An analysis of variance was then used to compare the size of the fluid cuff and the ratio of the cuff areas with the vessel areas.

Electron microscopic studies. The cardiac lobes were removed from the lungs of 12 dogs (4 controls, 3 HPPE, and 5 OAPE) just before freezing. Samples of lung tissue were excised from the ventral, mid, and dorsal surface of the lobe. Each sample was cut into 1-mm-thick blocks that were fixed in ice-cold $2.5 \%$ glutaraldehyde in $0.1 \mathrm{M}$ cacodylate buffer at $\mathrm{pH}$ 7.3. The tissue was postfixed in $1 \% \mathrm{OsO}_{4}$, stained en bloc with saturated uranyl acetate in distilled water, dehydrated in a graded ethanol series (propylene oxide), and then embedded in Spurr's resin (12). Thin sections were cut using glass knives on a Reichert ultracut ultramicrotome, mounted on 200-mesh copper grids, stained with uranyl acetate and lead salts, and viewed with a Philips 400 EM electron microscope. We examined and photographed the thin sections without prior knowledge of the treatment that the dog had received. A total of 910 photographs were taken, 580 of which were suitable for grading epithelia. They were examined by two observers who independently assigned a grade based on whether the epithelial cells were normal (grade I), showed evidence of vesiculation and/or abnormal cytoplasmic density and/or endomembrane hypertrophy (grade II), or showed evidence of epithelial cellular disruption with discontinuous epithelium and/or cells migrating through the epithelium (grade III).

Tracer studies. To minimize the cost of the fluorescein isothiocyanate (FITC)-labeled dextran tracer, these experiments were performed on New Zealand rabbits. 15 animals $(1.99 \pm 0.17 \mathrm{~kg}$ body weight, mean $\pm \mathrm{SD})$ were anesthetized with pentobarbital sodium $(8-12 \mathrm{mg} / \mathrm{kg}$ i.v.) and placed in the supine position so that the skin could be further anesthetized with $2 \%$ lidocaine hydrochloride before performing a tracheostomy. The animals were ventilated at $12-15 \mathrm{ml} / \mathrm{kg}$ tidal volume at a rate of $50-60$ breaths/min using a Harvard small animal ventilator. The left carotid artery was cannulated to measure systemic arterial pressure and a balloontipped catheter was placed in the aorta. A multiple side-holed catheter was placed in the right ventricle via the right internal jugular vein. Both ureters were then ligated. FITC-dextran $150(500 \mathrm{mg} / \mathrm{kg}$ of a $5 \%$ solution, average molecular weight 150,000, Sigma Chemical Co., St. Louis, MO) was injected intravenously. The animals were then randomly divided into control experiments $(n=5)$, OAPE experiments $(n=5)$ where oleic acid $0.1 \mathrm{mg} / \mathrm{kg}$ was injected into the right ventricle and HPPE experiments $(n=5)$ where pulmonary edema was produced by infusing a volume of $0.9 \%$ saline equivalent to $25 \%$ of body weight and inflating the aortic balloon to keep right ventricle systolic pressure in the range of 35-40 $\mathrm{cm} \mathrm{H}_{2} \mathrm{O}$.

In all of these studies, the animals were killed $2 \mathrm{~h}$ after the injection of the dextran by injecting a bolus of saturated $\mathrm{KCl}$ into the aortic root. The chest was then opened, the vessels ligated, and the lungs and heart removed as a single block. The lungs were inflated with air to a transpulmonary pressure of $20 \mathrm{~cm} \mathrm{H}_{2} \mathrm{O}$ and frozen over liquid nitrogen vapor. Random samples of the frozen tissue were fixed in cold $\left(-70^{\circ} \mathrm{C}\right) 4 \%$ glutaraldehyde in $100 \%$ ethanol for $3 \mathrm{~h}$. The blocks were dehydrated in ice-cold ethanol $(1 \mathrm{~h})$ and embedded in glycolmethacrylate (JB4, Polysciences Inc., Warrington, PA). 5- $\mu \mathrm{m}$ serial sections were cut, counterstained with Congo red $0.1 \%$ for $45 \mathrm{~s}$ at $60^{\circ} \mathrm{C}$ (Fisher Scientific Co., Forrest Lawn, NJ), then mounted in equal volumes of phosphate-buffered saline $(\mathrm{pH} \mathrm{8.6)}$ and glycerol containing the antioxidant $1-4$ diazobicyclo $[2,2,2]$ octane (Dabco, $100 \mathrm{mg} / \mathrm{ml}$ ) (Sigma Chemical Co.). Sections prepared in this way were examined in a single blind fashion by two independent observers using a Zeiss Universal epi-fluorescence microscope equipped with an HBO 50W illuminator, 450-490-nm exciter filter, FT510 nanometer beam splitter, and LP520 nanometer barrier. Each observer estimated the percentage of alveolar spaces containing FITCdextran and classified them into four groups depending on the degree of alveolar space filling (group I, 0-25\%, group II, 26-50\%, group III, $51-75 \%$, and group IV , 76-100\%). Bivariate prediction success and corrected contingency coefficients (11) were calculated to assess the reproducibility of the visual grading system. A chi-square analysis was performed using the pooled data from two observers to test for differences in alveolar fluorescence among the three groups (11). The remaining lung tissue was processed for the estimation of the wet and dry weights and a one-way analysis of variance was used to test for differences between the groups. The wet weight to dry weight ratios of the three groups were analyzed using one-way analysis of variance. 
In nine additional experiments, FITC-dextran $\mathbf{4 0}$ (average molecular weight 40,000 , Sigma Chemical Co.) was instilled in the airways via a tracheostomy in a $5 \%$ solution at a dose of $100 \mathrm{mg} / \mathrm{kg}$. $1-\mathrm{ml}$ blood samples were taken from the carotid artery before and at 15-min intervals after the FITC-dextran instillation. Blood withdrawn was replaced with an equal volume of $0.9 \%$ saline. In four of the nine animals, oleic acid $(0.1$ $\mathrm{mg} / \mathrm{kg}$ ) was injected into the right ventricle to produce OAPE at $1 \mathrm{~h}$ after the FITC-dextran $\mathbf{4 0}$ had been instilled in the airway. The remaining five animals served as controls. The blood samples were centrifuged at 1,500 rpm for $15 \mathrm{~min}$. Plasma fluorescence was detected using a spectrophotofluorometer (Aminco SPF-125, American Instrument Co., Silver Springs, MD). The excitation monochromator was set at $490 \mathrm{~nm}$ and the emission monochromator was set at $515 \mathrm{~nm}$ (13).

\section{Results}

\section{Physiological studies}

Table I contains the physiological data and shows that the initial conditions were the same for the three groups of dogs. After treatment, animals with both forms of edema showed comparable increases in the EVLW (group II, 8.2 \pm 1.9 grams per gram dry tissue (g/gdt); group III, $8.2 \pm 2.3 \mathrm{~g} / \mathrm{gdt}$ ). The EVLW in the control animals (group I) was $4.4 \pm 1.8 \mathrm{~g} / \mathrm{gdt}$, which is within the normal range for our laboratory. After $2 \mathrm{~h}$, group I showed a mild respiratory alkalosis but no other change. Group II showed a predictable increase in vascular pressures $(P<0.05)$. These animals also showed a fall in $\mathrm{Po}_{2}(P<0.05)$ and an increased Qs/Qt $(P<0.05)$ at the end of the 2 -h period. Group III showed no change in vascular pressues, a fall in cardiac output $(P<0.05)$, a fall in $\mathrm{PO}_{2}(P<0.05)$, and an increase in Qs/Qt $(P<0.05)$. The analysis of variance comparing the difference between the initial and final values showed that the increase in $\mathrm{Qs} / \mathrm{Qt}$ and the decrease in percent oxygen saturation (SAT) $\mathrm{O}_{2} \%$ was significantly greater in OAPE.

The regional blood flows at the beginning and end of the experiments are plotted against lung height in Fig. 1. With edema formation in HPPE, regional blood flow tended to increase, and this increase was seen to a greater degree in the upper regions of the lung (Fig. $1 b$ ). On the other hand, in OAPE, regional blood flow tended to decrease and remain uniformly distributed with respect to lung height (Fig. $1 c$ ). Fig. 2 shows that the amount of extravascular water present in the lung was not affected by gravity in any of the groups. Furthermore, the change in regional blood flow $\left(\Delta Q_{R}\right)$ that occurred during these experiments correlated poorly with the amount of extravascular lung water in that region (Table II).

\section{Morphological studies}

Light microscopy. Table III shows the results of the morphological studies on the distribution of edema fluid in the lungs under control, HPPE, and OAPE conditions. The chi-square analysis showed that both of the edematous groups differed from the control. Comparison of the two edema groups showed that OAPE was associated with more alveolar flooding and HPPE with more interstitial édema $(P<0.0005)$.

Table IV shows that the cuff area (mean \pm SE $\mathrm{mm}^{2} \times 10^{-2}$ ) averaged $0.17 \pm 0.02$ in control lungs, $0.30 \pm 0.02$ in HPPE, and $0.15 \pm 0.01$ in OAPE. The ratio of cuff area to vascular area was larger in HPPE $(2.26 \pm 0.45)$ than in OAPE $(0.45 \pm 0.09)$ or in the control lungs $(0.58 \pm 0.08)$. Both the cuff area and the ratio of cuff area to internal area were greater in HPPE $(P<0.001)$. There were also evidence of hemorrhage into the cuffs in OAPE but not in HPPE.

Electron microscopy. Fig. 3, $a$ and $b$, shows in a normal animal a patent capillary with a thin interstitial space, and intact epithelial and endothelial junctions (Fig. $3 \mathrm{~b}$ ). With moderate high pressure edema (Fig. $4 a$ ) the capillary is narrowed, the interstitium is thick (Fig. 4 b), and there is no alveolar flooding. With a similar amount of oleic acid-induced edema, on the other hand, there is alveolar flooding (Fig. $5 a$ ) with a tight junction that appears to be open (Fig. $5 b$ ). Furthermore, there is evidence of disruption of both alveolar epithelium (Fig. $6 a$ ) and endothelium, (Fig. $6 \mathrm{~b}$ ) in association with polymorphonuclear cell infiltration. The data from oleic acid-induced low pressure pulmonary edema also shows evidence of discharge of the lamellar bodies of type II cells (Fig. $6 c$ ) and the leakage of fibrinogen to form fibrin in the air space (Fig. $6 d$ ).

Table $\mathrm{V}$ shows a summary of the electron microscopic data. A total of 910 electron micrographs were examined; 580 of these were suitable for grading epithelium and in 526 there was agreement between the two observers as to the grade. This provided a contingency coefficient of $90 \%$ between the two observers. Table $\mathrm{V}$ shows the number of observations in each of the categories compared with the number of observations expected, which are shown in parentheses. In OAPE, the number of observations of

Table I. Physiological Data

\begin{tabular}{|c|c|c|c|c|c|c|}
\hline & \multicolumn{2}{|c|}{ Group I: Control } & \multicolumn{2}{|c|}{ Group II: High pressure edema } & \multicolumn{2}{|c|}{ Group III: Oleic acid-induced edema } \\
\hline & Initial & Final & Initial & Final & Initial & Final \\
\hline Ppa & $16 \pm 6$ & $16 \pm 6$ & $18 \pm 6$ & $43 \pm 6^{*} \ddagger$ & $15 \pm 4$ & $15 \pm 4$ \\
\hline Ppaw & $6 \pm 3$ & $8 \pm 5$ & $7 \pm 5$ & $33 \pm 7 * t$ & $7 \pm 2$ & $5 \pm 2$ \\
\hline $\mathrm{CO}$ & $2.5 \pm 0.8$ & $2.0 \pm 0.7$ & $2.5 \pm 0.4$ & $2.6 \pm 0.6$ & $2.4 \pm 0.8$ & $1.5 \pm 0.3 \ddagger$ \\
\hline PVR & $4.7 \pm 3.2$ & $5.2 \pm 1.9$ & $4.3 \pm 1.0$ & $3.5 \pm 1.1 \ddagger$ & $4.2 \pm 2.4$ & $7.6 \pm 5.2$ \\
\hline $\mathrm{pH}$ & $7.40 \pm 0.06$ & $7.46 \pm 0.13$ & $7.38 \pm 0.04$ & $7.23 \pm 0.07^{*} \ddagger$ & $7.37 \pm 0.02$ & $7.31 \pm 0.12^{*}$ \\
\hline $\mathrm{PCO}_{2} \mathbf{a}$ & $33 \pm 6$ & $26 \pm 7$ & $35 \pm 2$ & $30 \pm 6$ & $34 \pm 2$ & $34 \pm 14$ \\
\hline $\mathrm{PO}_{2} \mathrm{a}$ & $533 \pm 100$ & $516 \pm 91$ & $498 \pm 63$ & $270 \pm 144^{*} \ddagger$ & $510 \pm 59$ & $157 \pm 189^{*} \neq$ \\
\hline SAT $\mathrm{O}_{2} \%$ & 100 & 100 & 100 & $99 \pm 4$ & 100 & $88 \pm 13^{*}$ \\
\hline $\mathrm{Qs} / \mathrm{Qt}$ & $12 \pm 8$ & $16 \pm 10$ & $19 \pm 9$ & $39 \pm 15 \ddagger$ & $13 \pm 5$ & $48 \pm 29^{*} \ddagger$ \\
\hline EVLW & & $4.5 \pm 1.8$ & & $8.2 \pm 1.9^{*}$ & & $8.2 \pm 2.3^{*}$ \\
\hline
\end{tabular}

* $P<0.05$, analysis of variance comparing change between initial and final value edematous groups vs. change in control group except for EVLW, which compares actual values. $\ddagger P<0.05$, paired $t$ test initial vs. final values within a group. 

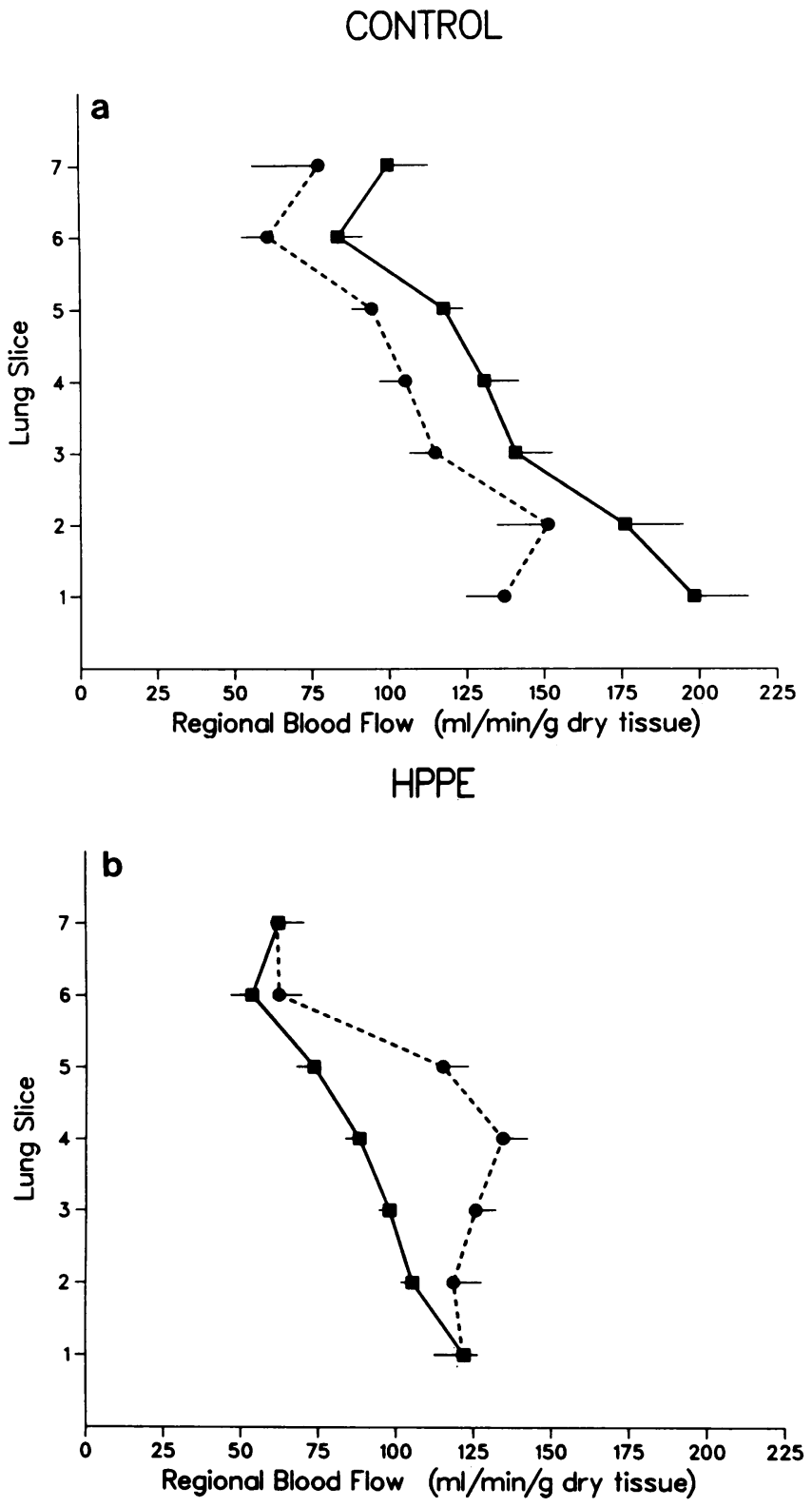

OAPE

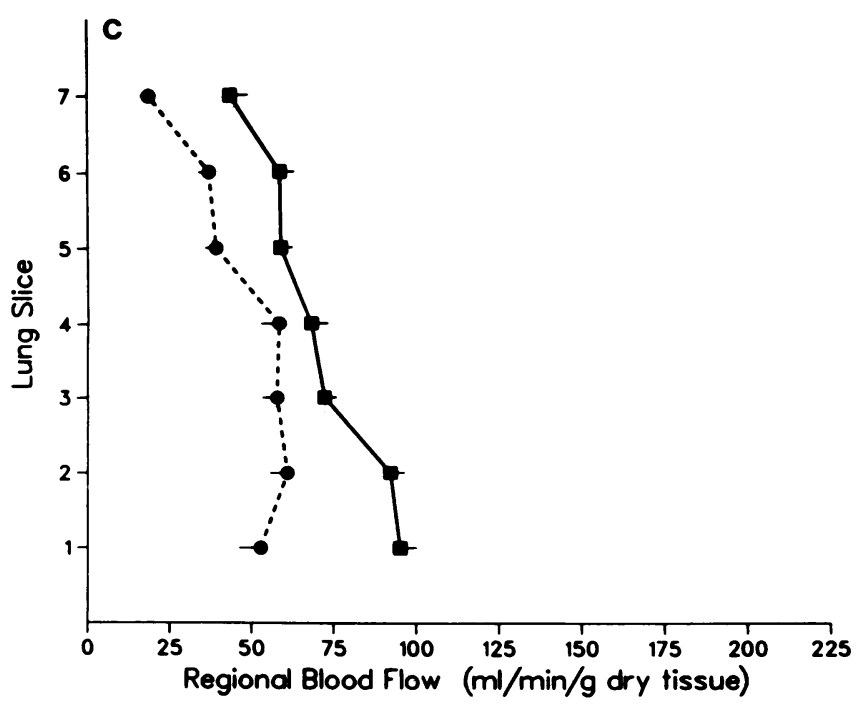

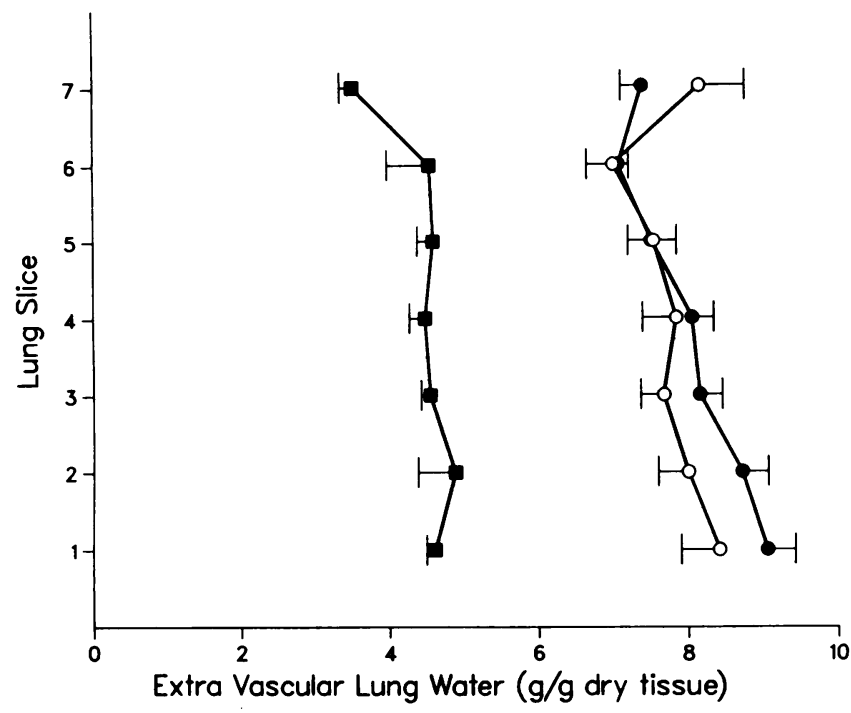

Figure 2. Shows the amount of extravascular lung water (mean \pm SE) at different lung heights in the control group $(\square)$. High pressure edema group $(O)$ and oleic acid edema group (๑).

moderate and severe disruption (i.e. grades 2 and 3) exceed the expected values, with 31 of 108 micrographs (28\%) showing evidence of damage. This was not true for control animals, where only $1.4 \%$ of 295 micrographs showed damage, or of the HPPE animals, where change was found in $2.4 \%$ of the 125 observed. Chi-square analysis (11) of this data showed greater epithelial disruption in OAPE $(P<0.0001)$.

\section{FITC tracer studies}

The data concerning the appearance of tracer in the alveolar spaces are shown in Table VI. The reproducibility of the visual grading system between the two observers was assessed and showed a bivariate prediction success of 0.872 and a corrected contingency coefficient of $88 \%$. The chi-square analysis performed in the data showed that the control and HPPE groups had equivalent degrees of alveolar fluorescence that were significantly less than the OAPE group $(P<0.01)$.

The appearance of FITC-dextran in the blood after it was placed on the lung surface (Fig. 7) shows that oleic acid injection was associated with an immediate rise in the plasma concentration of FITC-dextran. However, after this rapid rise the slope of two curves are quite similar until the end of the experiment.

Figure 1. (a) Regional blood flows (milliliters per minute per gram of blood-free dry lung) at different lung heights in the control group. There is no systematic redistribution of regional blood flow during the experiments. The solid line represents initial regional blood flows and the dotted line represents final regional blood flows after $2 \mathrm{~h} .(b) \mathrm{Re}-$ gional blood flows at different lung height before and after edema formation in the high pressure pulmonary edema group. The dotted line represents final regional blood flows after edema formation at $2 \mathrm{~h}$. There is an increase in blood flow to most regions and a shift in regional blood flows away from the dependent regions after edema formation. (c) Regional blood flows at different lung height before and after edema formation in the oleic acid-induced low pressure pulmonary edema group. The dotted line represents final regional blood flows after edema formation at $2 \mathrm{~h}$. There is a decrease in blood flow to most regions and no systematic shift of blood flow between regions after edema formation. The data points are represented by mean \pm SE. 
Table II. Relationship between Extravascular

Lung Water and Change in Blood Flow

\begin{tabular}{llll}
\hline & No. of samples & Slope & $\mathrm{R}^{2 *}$ \\
\hline Control & 222 & 0.015 & 0.38 \\
HPPE & 278 & 0.015 & 0.32 \\
OAPE & 276 & 0.030 & 0.49 \\
\hline
\end{tabular}

* Data show very weak relationship between the amount of extravascular water present and the change in blood flow that occurred in the individual samples of lung.

\section{Discussion}

The results of these experiments show that, for comparable amounts of extravascular lung water per gram of blood-free dry lung, the percent saturation of the arterial blood and the estimated shunt fractions are worse in oleic acid-induced low pressure pulmonary edema than in high pressure pulmonary edema (Table I). The observed difference in the shunt fractions, $48 \pm 29 \%$ in OAPE vs. $39 \pm 15 \%$ in HPPE (Table I), is likely an underestimate because Qs/Qt increases with $\mathrm{CO}$ (14) and the $\mathrm{CO}$ was lower in the OAPE group. This conclusion is supported by the two additional experiments where $\mathrm{CO}$ was increased in OAPE by opening arteriovenous shunts in the groins. In one of these experiments, $\mathrm{CO}$ increased from 2.26 liters/min to 2.96 liters/ $\min$ and Qs/Qt increased from 39.3 to $44.7 \%$. In the second, $\mathrm{CO}$ increased from $2.28 \mathrm{liters} / \mathrm{min}$ to $2.83 \mathrm{liters} / \mathrm{min}$, and Qs/ Qt increased from 54.8 to $61.9 \%$. Therefore, we conclude that the data in Table I provide a conservative estimate of the difference in shunt between HPPE and OAPE when similar amounts of edema are present.

The data from the light microscopic studies (Tables III and IV) show that oleic acid-induced low pressure pulmonary edema is associated with a greater degree of alveolar flooding (Table III) and that high pressure pulmonary edema is associated with more distension of the perivascular interstitial space (Table IV). These findings are consistent with the report by Zumsteg et al. (15) who found a pressure-independent pathway for alveolar flooding when low pressure pulmonary edema was produced by alloxan and a pressure-dependent pathway along the interstitium in high pressure pulmonary edema. Michel and his colleagues $(16,17)$ have shown that fluid accumulates around arteries in preference to veins and around large vessels in preference to small in both forms of edema. They did not comment on differences between interstitial and airspace edema in HPPE as opposed to LPPE. Previous studies (18) have shown that it is difficult to produce alveolar flooding in dogs by simply raising the pulmonary vascular pressures. Vreim et al. (3) showed that in high pressure pulmonary edema, with the extravascular lung water-to-dry weight ratio ranging from 6.6 to 15.7 , the alveolar flooding was less obvious. However, they had much less difficulty producing alveolar flooding in low pressure pulmonary edema (2).

A recent study by Julien et al. (19) shows that the amount of lung water present in low pressure pulmonary edema may have been underestimated by expressing it per gram of bloodfree dry tissue. This error would lead to our underestimate of the EVLW in oleic acid-induced pulmonary edema so that the excess alveolar flooding might be caused by the fact that more edema was present. In spite of this possible source of error, our data show (Table IV) that there was greater distension of the vascular cuffs in high pressure edema. This means that the edema was present in the interstitial space to a greater degree in HPPE than in OAPE, even though there may have been slightly more fluid present in OAPE.

The analysis of the electron microscopic data (Table V) show that oleic acid-induced edema was associated with more alveolar epithelial disruption than the control animals or those with HPPE. In these cases of OAPE we also found that there was discharge of type II (Fig. $6 c$ ) cells and fibrin deposits in the alveolar space (Fig. $6 d$ ). This contrasts sharply with the findings in high pressure pulmonary edema where the epithelium did not differ from the control studies (Table V).

The proposal that there is epithelial disruption in oleic acidinduced low pressure pulmonary edema leading to increased epithelial permeability and alveolar flooding is further substantiated by the dextran tracer studies performed in rabbits. These experiments show that FITC-dextran leaked from the blood to airspace to a greater degree in the oleic acid pulmonary edema group (Table VI). When FITC-dextran was measured in the blood after it was instilled into the airspace (Fig. 7), oleic acid injection caused an immediate increase in blood fluorescence. After this rapid rise the appearance of FITC-dextran in the blood was similar in OAPE and control. This observation is consistent with the findings of Jones et al. (20) and suggests a rapid control of the leak after the oleic acid damage. Recent studies by Dvorak et al. (21) have shown that extravascular coagulation can be initiated by large microvascular leaks, and the presence of fibrin on the alveolar surface (Fig. $6 d$ ) suggest that this occurred in OAPE. The observations from these studies and others $(20,21)$ therefore suggest that the fibrin deposition in alveoli that occurs as part of hyaline membrane formation may be a manifestation of extravascular coagulation that occurs in an attempt to control large microvascular leaks.

That FITC-dextran moved in both directions between the vasculature and the airspace (Table VI, Fig. 7) could only have occured as a result of a change in epithelial permeability. If al-

Table III. Light Microscopic Results on the Distribution of Extravascular Lung Water

\begin{tabular}{lllrrr}
\hline & EVLW & No. of slides examined & No edema & Interstitial edema & Alveolar flooding \\
\hline & $g / g$ dry lung & & & & \\
Control & $4.27 \pm 0.80$ & 25 & 20 & 4 & 1 \\
High pressure edema & $9.30 \pm 2.64$ & 60 & 21 & 21 & 18 \\
Oleic acid edema & $9.57 \pm 2.50$ & 64 & 4 & 9 & $52^{*}$
\end{tabular}

\footnotetext{
* The chi-square analysis (11) showed excess alveolar flooding in oleic acid-induced low pressure edema $(P<0.0005)$.
} 


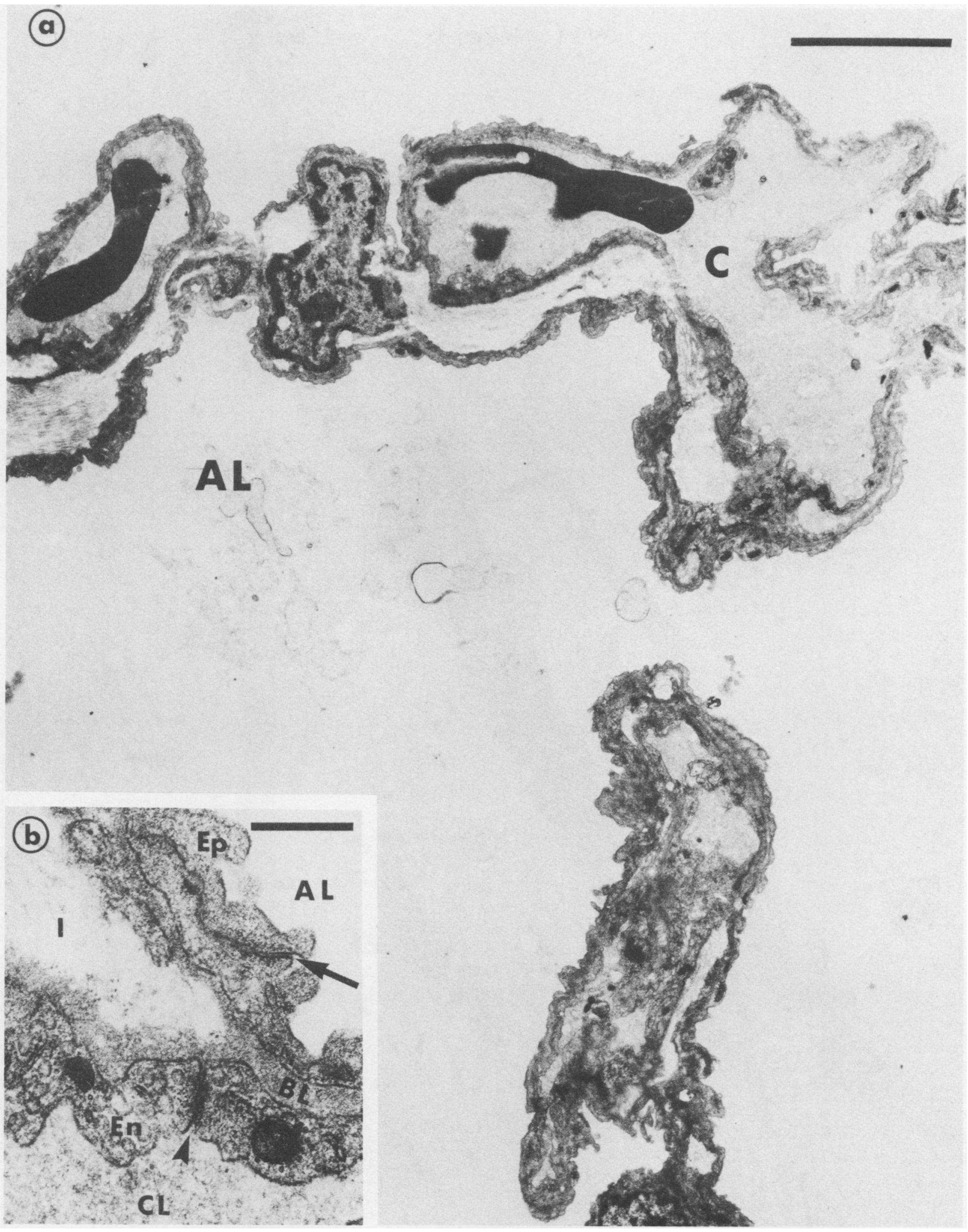

Figure 3. (a) Normal alveolar wall showing open capillaries (C) with both the thick and thin sides of the alveolar wall (AL, alveolar lumen). Scale bar, $5 \mu \mathrm{m}$. (b) Normal endothelial (En) and epithelial (Ep) tight junctions (arrowhead and arrow, respectively). Note interstitium (I) and basal lamina (BL). Scale bar, $0.5 \mu \mathrm{m}$.

veolar flooding is simply due to the loss of surfactant in OAPE, so that a negative surface pressure caused fluid to move into the alveolar space (22), the movement of FITC-dextran would have been unidirectional.
The conclusion that there is a difference in distribution of fluid in these two forms of lung edema is supported by the light microscopic, electron microscopic, and tracer studies. These show that in OAPE there is greater alveolar flooding (Tables III 


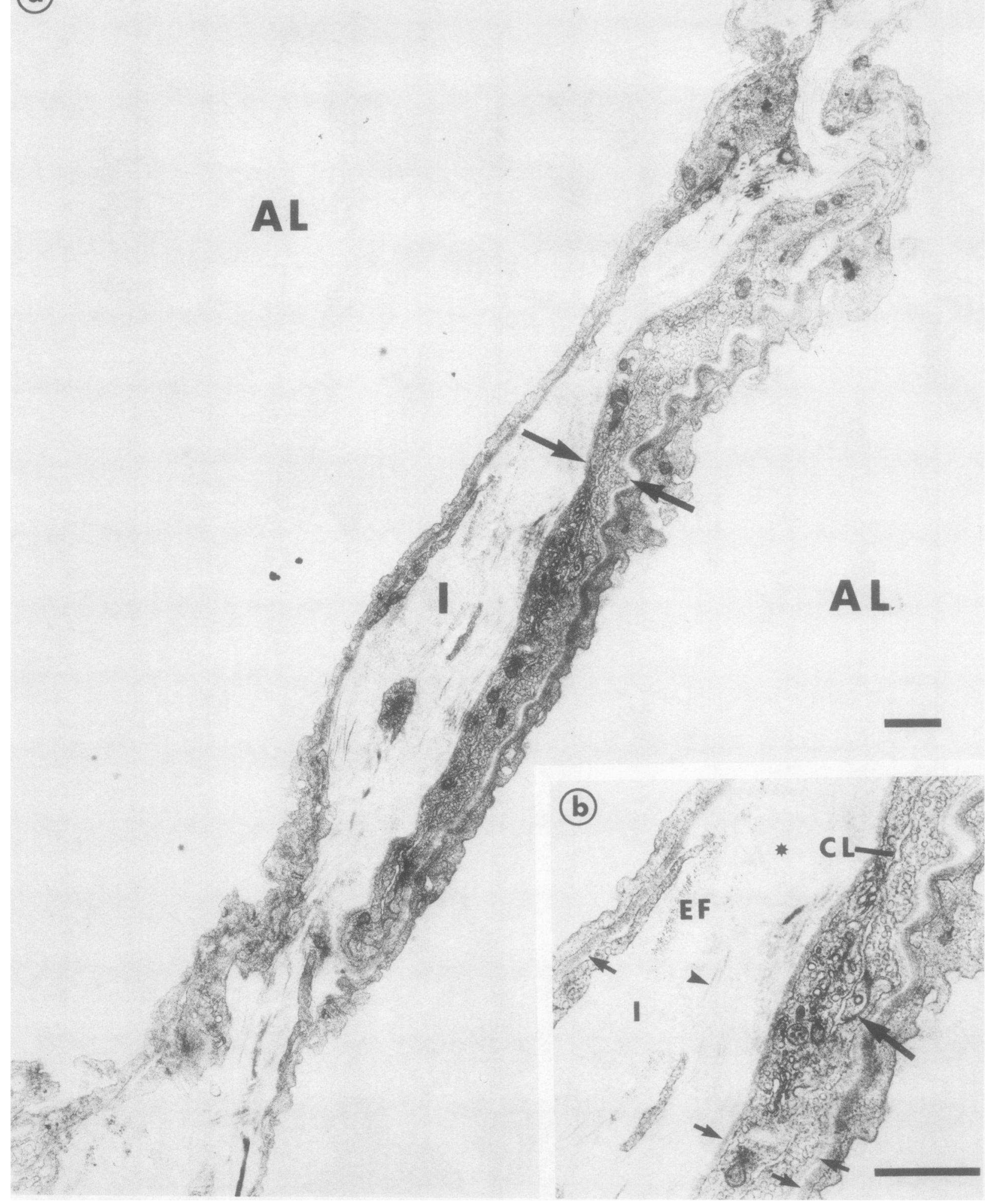

Figure 4. (a) High pressure edema without alveolar flooding where the interstitium (I) is thickened and the capillary (between arrows) is collapsed. Scale bar, $1 \mu \mathrm{m}$. (b) Widened interstitial space (I) between arrows on basal lamina of the endothelium and epithelium containing collagen (arrowhead) and elastic fibres (EF). The capillary lumen (CL) is collapsed and the endothelial tight junction (large arrow) is intact.

and IV), epithelial injury (Table V), and increased permeability from interstitium to airspace (Table VI) and airspace to interstitium (Fig. 7). The possibility that the epithelial injury is re- sponsible for the excess alveolar flooding and greater impairment of oxygenation in OAPE is attractive for several reasons. First, the difference in shunt between OAPE and HPPE (Table I) can- 


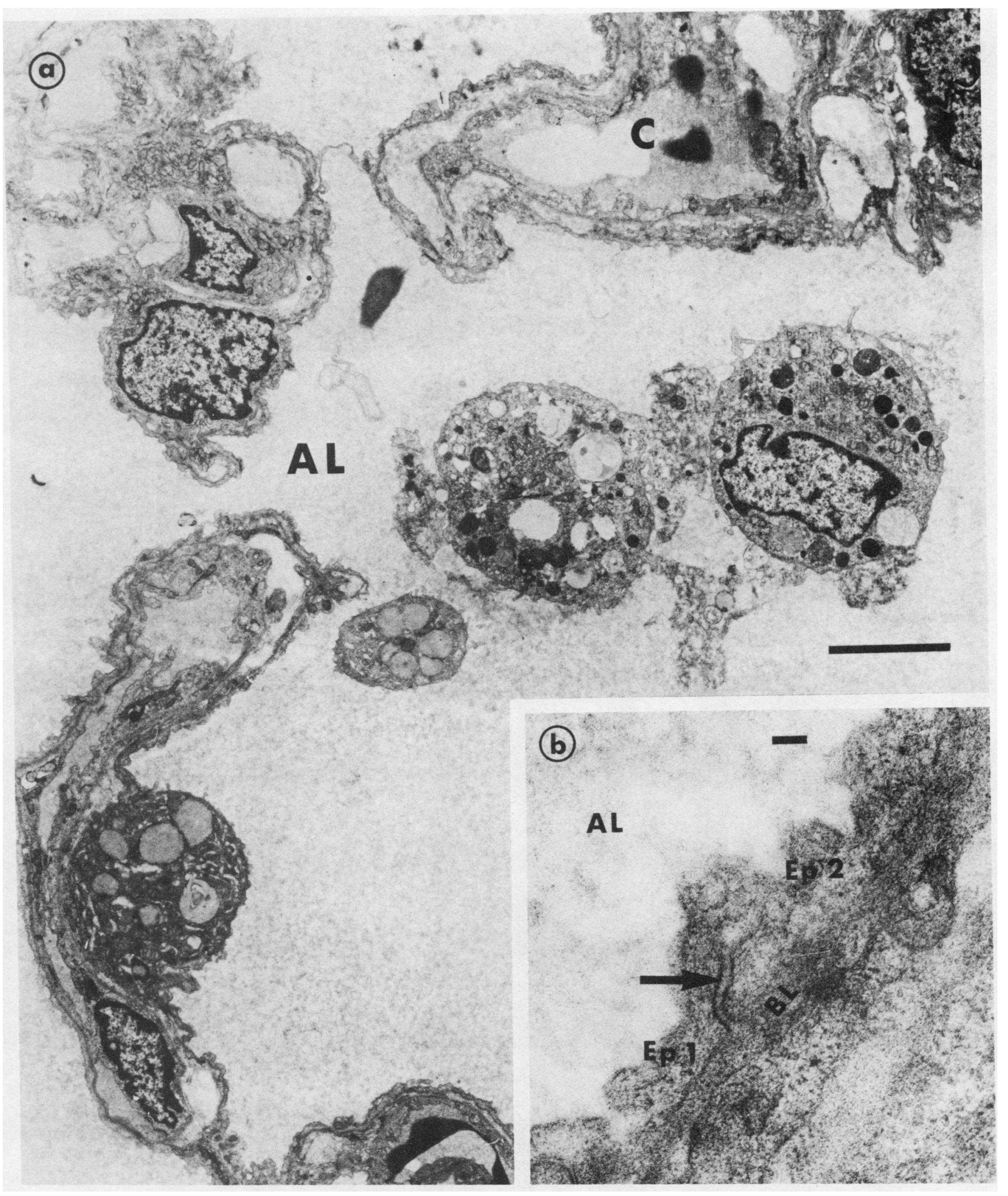

Figure 5. (a) Oleic acid-induced low pressure edema with alveolar flooding showing fluid and marcrophages in the alveolar lumen (AL). Scale bar, $5 \mu \mathrm{m}$. (b) Alveolar wall with a tight junction (arrow) between epithelial cells that appear to be open (Ep 1 and Ep 2). BL, basal lamina; scale bar, $0.1 \mu \mathrm{m}$.

not be explained by differences in the regional blood flow. The change in regional blood flow after edema formation was not affected by the amount of extravascular lung water that accu- mulated in the region in either OAPE or HPPE (Table II). This implies that the difference in oxygenation in the two forms of edema is more likely due to the difference in airspace flooding 


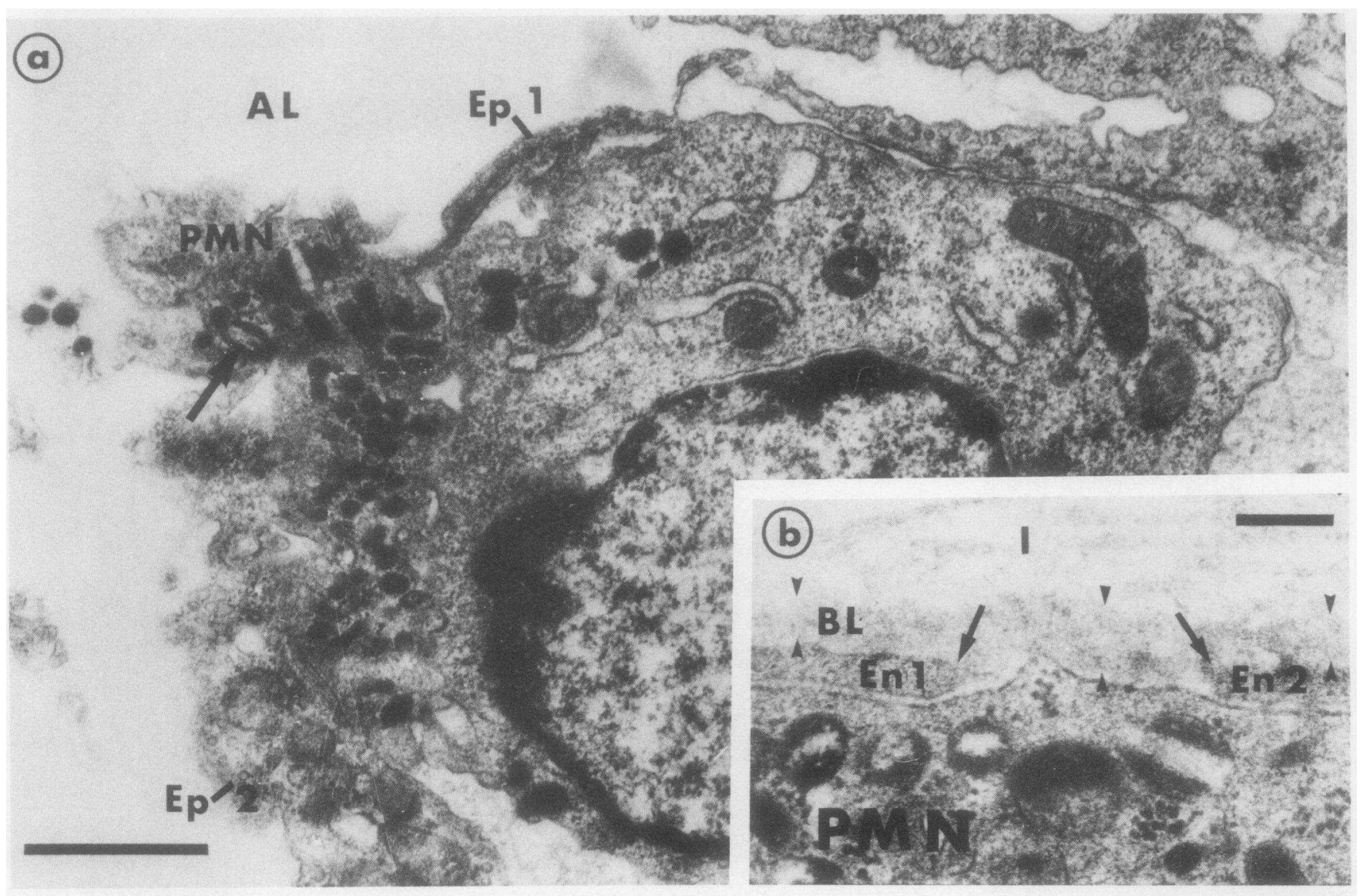

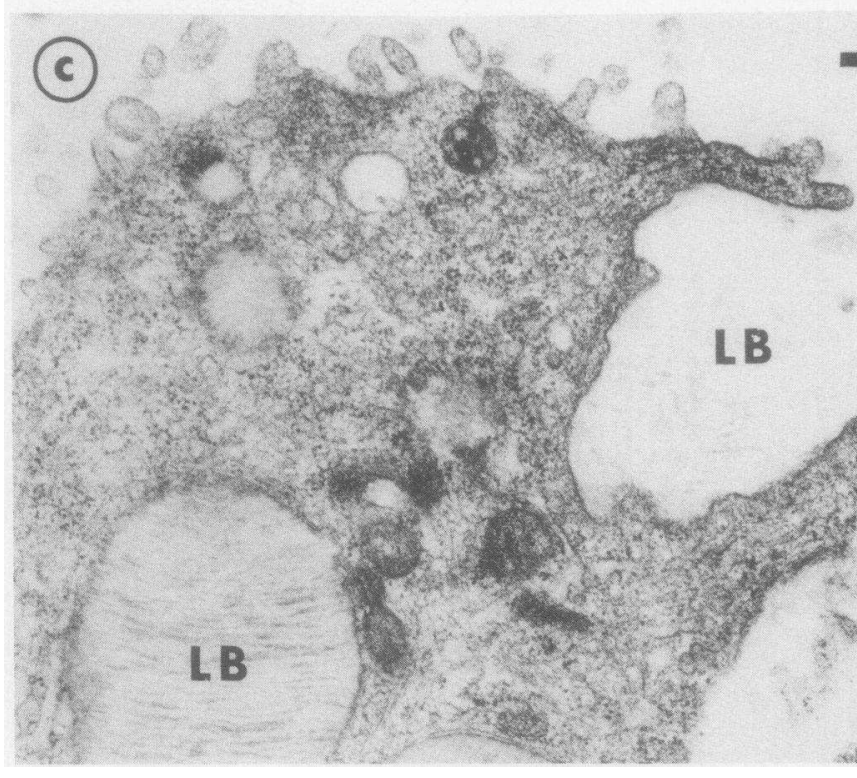

Figure 6. (a) Oleic acid-induced edema. Note a polymorphonuclear cell (PMN) entering the alveolar lumen (AL) through an epithelial disruption (Ep 1 and Ep 2). Scale bar, $1 \mu \mathrm{m}$. (b) Disruption of the endothelium (note arrows outlining space between endothelial cells En 1

(Table III). An increase in airspace fluid would lead to increased alveolar instability and collapse (23), which would reduce the surface area available for gas exchange. Furthermore, as oxygen dissolves poorly in water and $\mathrm{O}_{2}$ diffuses 300,000 times slower through liquid than gas (24), the oxygen content in the flooded alveoli would be rapidly depleted unless the fluid was in close and constant contact with a gaseous reservoir. In addition, the pressure perfusing the vascular bed is lower in OAPE, so that capillary blood volume will be smaller and diffusing capacity will be lower than the HPPE (25).

Dantzker (26) has analyzed the ventilation-perfusion (V/Q) distributions in patients with adult respiratory distress syndrome 
Table IV. Light Microscopic Results on the Distribution of Lung Water Around the Vascular Cufss

\begin{tabular}{lccc}
\hline & Control & HPPE & OAPE \\
\hline No. of animals & 1 & 2 & 2 \\
No. of vessels & 220 & 871 & 745 \\
Cuff area $\pm S E$ & & & \\
$\quad\left(\times 10^{-2} \mathrm{~mm}^{2}\right)$ & $0.17 \pm 0.02$ & $0.30 \pm 0.02^{*}$ & $0.15 \pm 0.01$ \\
Cuff/internal area & $0.58 \pm 0.08$ & $2.26 \pm 0.45^{*}$ & $0.45 \pm 0.09$ \\
Intracuff hemorrhage & 0 & 3 & 30 \\
EVLW (mean \pm SD) & $4.27 \pm 0.80$ & $9.30 \pm 2.64$ & $9.57 \pm 2.50$
\end{tabular}

* The analyses of variance showed larger cuff areas and a greater cuff to internal vascular area ratio in HPPE $(P<0.001)$.

(ARDS) by inert gas technique and reported that the gas exchange units under such conditions exist either as normal units or units with very low $V / Q$ ratio. Assuming that oleic acid-induced edema is a realistic model of clinical ARDS, our data suggest that the units with very low $V / Q$ ratios might be accounted for by the excess flooding of alveoli. The fact that gas exchange was less effected in HPPE could be explained by the fact that the fluid was in the interstitium rather than the airspace, and increased thickness of the interstitium does not significantly impair oxygen diffusion (27).

The data reported here are consistent with the results of Noble and his associates $(28,29)$, who have shown that alveolar to

Table V. Electron Microscopic Results

\begin{tabular}{lllcl}
\hline & \multicolumn{2}{l}{ Number of samples } & & \\
\cline { 2 - 4 } Group & Grade $1^{*}$ & Grade 2 & Grade 3 & Total \\
\hline Control & $291(275) \ddagger$ & $2(5)$ & $2(5)$ & 295 \\
OAPE & $77(101)$ & $6(2)$ & $25(5) \S$ & 108 \\
HPPE & $122(114)$ & $1(2)$ & $0(7)$ & 125 \\
Total & 490 & 9 & 27 & 526 \\
\hline
\end{tabular}

* Grade I, normal epithelium; grade II, epithelial abnormality; grade III, epithelial disruption (see Methods for further description). $¥$ The numbers in parentheses represent the number of samples expected in each category if they were randomly distributed among the three groups.

$\S$ Chi-square analysis showed greater epithelial disruption in OAPE ( $P$ $<0.0001$ ).

Table VI. FITC Dextran in Alveolar Space

\begin{tabular}{llll}
\hline & \multicolumn{2}{l}{ Number of samples } & \\
\cline { 2 - 4 } & Control & HPPE & OAPE \\
\hline None & 15 & 13 & 6 \\
$1-25$ & 5 & 7 & 5 \\
$26-50 \%$ & 0 & 0 & 6 \\
$51-75 \%$ & 0 & 0 & 3 \\
$76-100 \%$ & 0 & 0 & 0 \\
Present/total & $5 / 20$ & $7 / 20$ & $14 / 20^{*}$
\end{tabular}

* Chi-square analysis showed significantly greater alveolar fluorescence in the OAPE group compared with both the control and HPPE groups $(P=0.01)$.

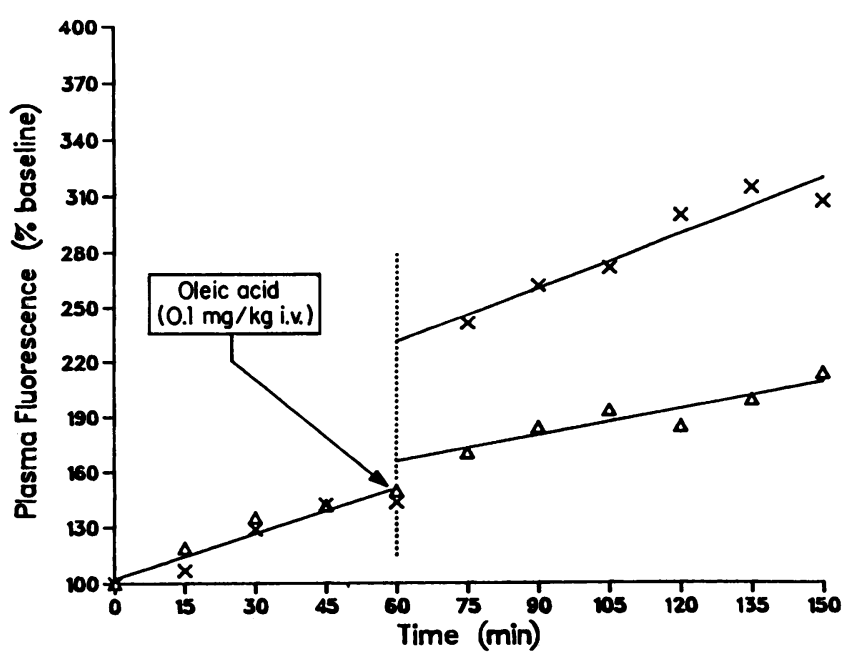

Figure 7. Shows the mean change in plasma fluorescence caused by the instillation of FITC-labeled dextran down the airway at time zero in two groups of animals. When oleic acid was injected intravenously in one group of animals there was a sharp rise in plasma fluoresence, which indicated a rapid increase in permeability.

arterial (A-a) gradient remains low in interstitial edema but increases markedly when alveolar edema develops. This increase in alveolar to arterial (A-a) gradient was found to be out of proportion to the increase in the amounts of lung water present when interstitial edema progressed to alveolar flooding. The observation that abnormal gas exchange correlated better with the amount of alveolar flooding than the amount of edema present could help to explain the data of Brigham et al. (30). They found that the impairment of oxygenation correlated poorly with the amount of extravascular lung water. The fact that the methodology they used would not allow them to determine whether the excess water was in the airspace or interstitium could explain their finding.

These results show that the epithelial injury occurs in OAPE and that it represents a critical difference between lungs with OAPE and HPPE when similar amounts of edema are present. This epithelial disruption leads to an increased number of fluidfilled alveoli which have preserved regional blood flow resulting in regions with very low $\mathrm{V} / \mathrm{Q}$. The lack of epithelial disruption in HPPE allows more of the extravascular lung water to be stored in the interstitium, where it has less effect on gas exchange.

\section{Acknowledgments}

The authors thank E. M. Baile and A. MacKenzie for their technical assistance and Lee Kowk for her help in preparing the manuscript.

The work was supported by the British Columbia Heart Foundation and the Medical Research Council (MRC) of Canada (grant 4219 to Dr. Montaner). Dr. Evans and Dr. Walker were supported by the British Columbia Lung Association, and Dr. Mullen by a postdoctoral Fellowship from the MRC of Canada. Dr. Tsang was supported by the B. C. Health Care Research Foundation.

\section{References}

1. Staub, N. C., H. Nagano, and M. L. Pearce. 1967. Pulmonary edema in dogs, especially the sequence of fluid accumulation in the lungs. J. Appl. Physiol. 22:227-240.

2. Vreim, C. E., and N. C. Staub. 1976. Protein composition of lung fluids in acute alloxan edema in dogs. Am. J. Physiol. 230:376-379. 
3. Vreim, C. E., P. D. Snashall, and N. C. Staub. 1976. Protein composition of lung fluids in anaesthetized dogs with acute cardiogenic edema. Am. J. Physiol. 231:1466-1469.

4. Staub, N. C. Pulmonary edema. 1974. Physiol. Rev. 54:678-721.

5. Schoene, R. B., H. T. Robertson, D. R. Thorning, S. C. Springmeyer, M. P. Hlastala, and I. W. Cheney. 1984. Pathophysiological patterns of resolution from acute oleic acid lung injury in the dog. J. Appl. Physiol. 56(2):472-481.

6. Hogg, J. C., P. Holst, P. Corry, F. Ruff, E. Honsley, and E. Morris. 1971. Effect of regional lung expansion and body position on pulmonary perfusion in dogs. J. Appl. Physiol. 31:97-101.

7. Derks, C. M., and D. Jacobovitz-Derks. 1977. Embolic pneumopathy induced by oleic acid. Am. J. Pathol. 87:143-151.

8. Gray, S. J., and K. Sperling. 1950. The tagging of red cells and plasma protein with radioactive chromium. J. Clin. Invest. 29:16041613.

9. Mcllroy, M. B. 1965. Pulmonary Shunts Handbook of Physiology, Section III, Vol. 2. W. O. Lenn and H. Rahn, editors. American Physiological Society, Washington, D.C. 65:1519-1524.

10. Baile, E. M., P. D. Pare, R. W. Dahlby, and J. C. Hogg. 1979. Regional distribution of extra vascular lung water and hematocrit in the lung. J. Appl. Physiol. 46:937-942.

11. Sachs, L. 1982. Applied Statistics: A Handbook of Technique. Springer Series in Statistics, New York (English translation). 6:462-493.

12. Spur, R. A. 1969. A low viscosity epoxy resin embedding medium for electron microscopy. J. Ultrastruct. Res. 26:31-43.

13. Takada, K., M. Yamamoto, and S. Masada. 1978. Evidence for the pulmonary absorption of fluorescent labelled macromolecular compounds. J. Pharm. Dyn. 1:281-287.

14. Lynch, J. P., J. G. Mhyre, and D. R. Dantzker. 1979. Influence of cardiac output on the intrapulmonary shunt. J. Appl. Physiol. 46: 315-321.

15. Zemsteg, T. A., A. M. Havill, and M. H. Gee. 1982. Relationships among lung extravascular fluid components with alveolar flooding. $J$. Appl. Physiol. 53:267-271.

16. Michel, R. P., T. S. H. Akinn, T. T. Smith, and R. S. Paulsen. 1983. Quantitative morphology of permeability edema in dogs induced by napthylthiouria. Lab. Invest. 149:412-419.

17. Michel, R. P., T. T. Smith, S. Meterissian, and R. S. Paulsen.
1984. Distribution of interstitial fluid in hydrostatic lung edema: a morphometric study. Am. Rev. Respir. Dis. 129:A347 (Abstr.)

18. Guyton, A. C., and A. W. Lindsey. 1959. Effect of elevated left atrial pressure and decreased plasma protein concentration on the development of pulmonary edema. Circ. Res. 7:649-657.

19. Julien, M., M. R. Flick, J. M. Hoeffel, and J. F. Murray. 1984. Accurate reference measurement of postmortem lung water. J. Appl. Physiol. 56:248-253.

20. Jones, J. G., B. D. Minty, J. M. Beeley, D. Royston, J. Crow, and R. F. Grossman. 1982. Pulmonary epithelial permeability is immediately increased after embolization with oleic acid but not with neutral fat. Thorax. 37:169-174.

21. Dvorak, H. F., D. R. Senger, A. M. Dvorak, V. S. Harvey, and J. McDonagh. 1985. Regulation of extravascular coagulation by microvascular permeability. Science (Wash. DC). 227:1059-1061.

22. Avery, M. E., and J. Mead. 1959. Surface properties in relation to atelectasis and hyaline membrane disease. Am. J. Dis. Child. 97:517523.

23. Bachofen, H., P. Gehr, and E. R. Weibel. 1979. Alteration of mechanical properties and morphology in excised rabbit lungs rinsed with a detergent. J. Appl. Physiol. 47:1002-1010.

24. Weibel, E. R. 1984. The Pathway for Oxygen: Structure and Function in the Mammalian Respiratory System. Harvard University Press, Cambridge. 1:12-13.

25. Bachofen, H., J. Weber, D. Wangensteen, and E. R. Weibel. 1983. Morphometric estimates of diffusing capacity in lungs fixed under Zone II and Zone III conditions. Respir. Physiol. 52:41-52.

26. Dantzker, D. R., J. P. Lynch, and J. G. Weg. 1980. Depression of cardiac output is a mechanism of shunt reduction in the therapy of acute respiratory failure. Chest. 77:636-642.

27. McHardy, G. J. R. 1972. Diffusing capacity and pulmonary gas exchange. Br. J. Dis. Chest. 66:1-20.

28. Noble, W. H., K. Kovacs, and J. C. Kay. 1974. Fine structure changes in hemodynamic pulmonary edema. Can. Anaesth. Soc. J. 21: 275-284.

29. Noble, W. H., J. C. Kay, and J. Obdrzalek. 1975. Lung mechanics in hypervolemic pulmonary edema. J. Appl. Physiol. 38:681-687.

30. Brigham, K. L., K. Kariman, T. Harris, J. R. Snapper, G. R. Bernard, and S. L. Young. 1983. Correlation of oxygenation with vascular permeability-surface area but not with lung water in humans with acute respiratory failure and pulmonary edema. J. Clin. Invest. 72:339-349. 